\title{
A COMPARATIVE STUDY OF FINE NEEDLE ASPIRATION CYTOLOGY AND OPEN BIOPSY IN THE DIAGNOSIS OF CERVICAL LYMPHADENOPATHY IN RURAL TERTIARY CENTRE
}

\author{
Karunamoorthy Rajachidambaram1, Thiagraj Natraj² \\ ${ }^{1}$ Associate Professor, Department of General Surgery, Chennai Medical College Hospital \& Research Centre, Irungalur, Trichy. \\ ${ }^{2}$ Assistant Professor, Department of General Surgery, Karpagam Faculty of Medical Sciences \& Research, Coimbatore.
}

\section{ABSTRACT}

Lymphadenopathy is a very common clinical manifestation of many diseases, defined as an abnormality in the size or character of lymph nodes, caused by the invasion or propagation of either inflammatory cells or neoplastic cells into the node. The clinical examination may be inaccurate in differentiating benign from neoplastic enlargement of the lymph node. Surgical excision of a palpable peripheral lymph node is relatively simple; it does require anaesthesia, strict sterility and theatre time and it leaves behind a scar. Fine needle aspiration cytology offers the advantages of an immediate with little cost and trauma. The present study is being undertaken to evaluate its accuracy in the diagnosis of tuberculosis and malignancy of the cervical lymph nodes.

\section{AIM AND OBJECTIVE}

To assess the usefulness of FNAC in the diagnosing cervical lymph node enlargement and cytological features of common pathological conditions affecting the lymph nodes and to evaluate the diagnostic accuracy of FNAC and open biopsy in cervical lymphadenopathy.

\section{MATERIALS AND METHODS}

A total of 100 patients were subjected for the study at $\mathrm{CMCH}$ and $\mathrm{RC}$, Irungalur. FNAC was performed under aseptic precaution of the cervical lymph nodes using a 22G needle and HPE correlation was obtained for these patients remaining. These patients were subjected to surgical excision for radical dissection and the HPE correlation for specimen was obtained.

\section{RESULTS}

The result shows that the age wise distribution of patient with benign lesions were within 21-25 age groups and those with malignant lesion were in 31-50 age groups. Among the incidence of male-to-female ratio was 107:93. The duration of symptom was $<6$ months ( $<3 \mathrm{~cm}$ 50\%/3-6 months 45\%). The gross morphology shows that TB adenitis patient with positive matting nodes were 18 out of 25 patients were positive for matted nodes (94.47\%). According to AJCC cancer staging the size of the nodes were assessed, which shows most of the nodes $<3 \mathrm{~cm}(84 \%)$. The result shows comparison between FNAC and HPE in patient who had lymphoma and 83 out of 100 were +ve for FNAC and all patient +ve for HPE.

\section{CONCLUSION}

This study demonstrates that fine needle aspiration is a safe accurate and valuable tool in the evaluation of cervical lymphadenopathy.(1) Analysis shows that HPE is gold standard for evaluation of cervical node in neck for accuracy in approaching $100 \%$. FNAC analysis shows that accuracy is $95 \%$, since HPE diagnosis is invasive procedure. FNA is better alternate to HPE for malignancy and occult primary for lymph node and benign lesion, it is complimentary. So we recommend that reliable supplement, however, role of HPE is absolute.

\section{KEYWORDS}

Lymphadenopathy, Diagnostic Efficacy, Fine Needle Aspiration Cytology and Histopathological Examination.

HOW TO CITE THIS ARTICLE: Rajachidambaram K, Natraj T. A comparative study of fine needle aspiration cytology and open biopsy in the diagnosis of cervical lymphadenopathy in rural tertiary centre. J. Evolution Med. Dent. Sci. 2016;5(35):1979-1983,

DOI: $10.14260 /$ jemds/2016/468

\section{INTRODUCTION}

In the diagnosis of tuberculosis and malignancy of the cervical lymph nodes, fine needle aspiration cytology is the study of cells obtained by a small gauge needle generally with a vacuum system provided by an airtight syringe. This technique has been in use for studying pathological lymph node enlargement since.

Financial or Other, Competing Interest: None.

Submission 10-03-2016, Peer Review 22-03-2016,

Acceptance 25-03-2016, Published 30-04-2016.

Corresponding Author:

Dr. Karunamoorthy Rajachidambaram,

93G/5A Chidambaram Nagar,

Near New Bus stand,

Perambalur-621212.

E-mail: drkrajachidambaram@gmail.com

DOI: 10.14260/jemds/2016/468
Lymph node enlargement may be due to a variety of underlying diseases, both benign and malignant. The clinical examination may be inaccurate in differentiating benign from neoplastic enlargement of the lymph node. Although surgical excision of a palpable peripheral lymph node is relatively simple, it does require anaesthesia, strict sterility and theatre time and it leaves behind a scar.

Fine needle aspiration cytology offers the advantages of an immediate, although not always specific diagnosis with little cost and trauma.

The primary purpose of fine needle aspiration biopsy of an abnormal peripheral lymph node is to decide on further course of treatment whether medical, surgical or observation.(1)

The alternatives are to just observe the patient to carry out further investigations or try a course of conservative treatment for example with antibiotics. Obviously, there are 
practical and psychological advantages if this decision can be made within a day.

The accuracy of diagnosis has improved considerably over the past few years. Therefore, FNAC has got a definite role in the initial evaluation of all patients with peripheral lymphadenopathy. The present study is being undertaken to evaluate its accuracy.

\section{AIM AND OBJECTIVE}

\section{The purpose of the study is}

1. To assess the usefulness of FNAC in the diagnosis of cervical lymph node enlargement.

2. To study the cytological features of common pathological conditions affecting the lymph nodes, viz. tuberculosis, and lymphoma and secondary malignancy.

3. To evaluate the diagnostic accuracy of FNAC and open biopsy in cervical lymphadenopathy.

\section{Methodology and Protocol Followed}

This is a prospective study period extending from July 2013 to June 2015 in Chennai Medical College Hospital. The patients presenting with cervical lymphadenopathy for whom open biopsy was planned were subjected to FNAC. At the time of open Biopsy smear were taken and fixed. Following final histopathology report, the FNAC and HPE reports were compared and analysed.

\section{Review of Literature}

"Many observations have been made by morphologists and pathologists interested in normal and morbid cytology and its diagnostic possibilities. This impressive volume of work did not reach a stage of recognition for decades until finally its cumulative force strengthened by the impact of new contributions caused a break in the dam of inertia and scepticism that blocked its progressive course." Papanicolaou (1883-1962) - the father of exfoliative cytology. ${ }^{3}$ Kun. $^{7}$ introduced needle aspiration in 1841. Erichson (1853) adopted this method of using an exploring needle to withdraw cells from tumours for cytological diagnosis. It was, however, only after Papanicoloau's basic discovery in 1928 of the usefulness of exfoliated cells in the diagnosis of carcinoma that the cytological diagnosis of tumour became popular. Fine needle aspiration of lymph nodes has been around for quite a long time. In 1904 Greig and Gray.4,5 isolated trypanosomes by aspirating lymph nodes in patients with sleeping sickness.

The technique has subsequently been used to study both benign and malignant diseases. However, the procedure did not gain wide acceptance in medicine at that time. In the 1930s, Memorial Sloan Kettering.2,7 rediscovered the utility of needle biopsy of head and neck masses. The use of large-bore needles at that time led to frequent complications, one of which was occasional seedling of the tumour along the biopsy tract. The frequent morbidity associated with this procedure prevented widespread acceptance of this technique in other centres of America. A resurgence of FNA occurred in the 1950s, led by physicians in Sweden. FNA was commonly used for cytologic examination of metastatic lesions in the neck with excellent results. Since then FNA of solitary neck masses has become a well-accepted, safe, and cost-effective procedure in the diagnosis of neck masses.
Forkner (1927). ${ }^{9}$, Martin and Ellis (1930).8,10,11 and Stewart (1933) were the pioneers in using fine needle aspiration for lymph node enlargement. This procedure gradually gained momentum through the efforts of Franzen, Zajicek. ${ }^{6}$ and Sodernstorm in Sweden and now it has become an extremely well documented technique in the diagnosis of palpable/localized masses. In India the technique was first introduced at PGI, Chandigarh, in the early seventies followed by AIIMS, New Delhi, in the mid-seventies.8,12 The assumption that aspiration biopsy spreads malignancy appears theoretical rather than actual. Zajicek and Engzell. ${ }^{16}$ found no clinical evidence of tumour dissemination along the needle tract after a long follow-up of lymph node FNAC (1971).

\section{RESULT}

- A total of 100 patients were subjected to FNAC of cervical lymph nodes using a $22 \mathrm{G}$ needle on a $10 \mathrm{~mL}$ syringe. HPE correlation has obtained in 73 patients, since remaining 27 patients were diagnosed as non-specific lymphadenitis. They were treated with antibiotics and followed up in the outpatient department. The 73 patients were subjected to surgical excision routinely as part of the study or a part of radical dissection and HPE correlation obtained.

- Breakup of the cases according to the histology is given in the Table, which follow: -

- $\quad$ The size of the nodes, which were sampled range from 2$6 \mathrm{~cm}$. No complications occurred in the series. All inadequate and inconclusive aspirations were repeated.

\section{Metastatic Lymphadenopathy}

- Of the 33 cases examined a total of 30 cases were positive, while 3 were false negative. The diagnostic accuracy was $91 \%$, the type was not specified in 4 smears and only a diagnosis of positive for malignant cells was given. Also the correlation was inaccurate in 2 cases where adenocarcinoma and squamous cell carcinoma were reported as anaplastic carcinoma.

- Among the 3 false negatives, 2 cases on histopathological examination showed partial replacement of the nodes by malignant cell. The samples were therefore not representative. The other one was a cystic metastasis from occult papillary carcinoma of thyroid reported as a benign cystic lesion.

\section{Tuberculosis}

- Of the 25 cases identified by histopathology correlation with FNAC was obtained in 22 cases. The diagnostic accuracy.(2) was $88 \%$.

- In 2 cases, a non-specific diagnosis of granulomatous adenitis was reported.

- Among the false negatives where a clinical suspicion was entertained (supplemented with positive Mantoux and history of exposure), two were reported as chronic nonspecific adenitis and in one adequate smear could not be obtained due to the presence of necrosis. 
Break-Up Cases According to Histology

\begin{tabular}{|c|c|c|c|}
\hline Disease & $\begin{array}{c}\text { HPE } \\
\text { +ve }\end{array}$ & $\begin{array}{c}\text { FNAC } \\
\text { +ve }\end{array}$ & $\mathbf{\%}$ \\
\hline Tuberculous Lymphadenitis & 25 & 22 & $58 \%$ \\
\hline Malignant Disease & 48 & 43 & $60 \%$ \\
\hline Metastatic adenocarcinoma & 5 & 3 & $60 \%$ \\
\hline $\begin{array}{c}\text { Metastatic squamous cell Ca. } \\
\text { (Primary known) }\end{array}$ & 9 & 9 & $100 \%$ \\
\hline $\begin{array}{c}\text { Metastatic squamous cell Ca. } \\
\text { (Unknown primary) }\end{array}$ & 6 & 6 & $100 \%$ \\
\hline Metastatic thyroid carcinoma & 6 & 5 & $83 \%$ \\
\hline $\begin{array}{c}\text { Metastatic anaplastic } \\
\text { carcinoma }\end{array}$ & 7 & 7 & $100 \%$ \\
\hline Reactive hyperplasia & & 27 & $100 \%$ \\
\hline
\end{tabular}

\section{DISCUSSION}

- $\quad$ FNAC is an effective way to diagnose the pathological process underlying lymphadenopathy.(3)

- The diagnostic accuracy in various series ranges from $85 \%$ to $100 \%$. Diagnostic accuracy in this study was $88 \%$ for benign lesion and $89 \%$ for malignancy. It is comparable with various studies. ${ }^{25,26,27,28}$

- The high rate of accuracy and its simplicity here resulted in FNAC occupying an important place in the initial evaluation of cervical lymphadenopathy. It is still not considered as an alternative to histopathological examination of the node.

- 'An enlarged lymph node should never be excised as the first or even an early step in diagnosis' - Hayes Martin. This is especially true in the case of metastatic malignancy with unknown primary.(4) were in it may be detrimental for a number of reasons including.

- Local and possibly general spread of the disease.

- A false sense of security for the patient, who feels that the lump has been removed.

- Compromise of adequate radical dissection due to improperly placed incision.

- Wound infection delaying definitive surgery.

- The advantage of FNAC lies in facilitating further workup in the search of an occult primary tumour.(5) The cytological patterns give clues to the site of the primary tumour.

- Only when the primary cannot be found despite the evidence provided by FNAC and supplementary investigations and when this information is likely to be of therapeutic importance is a surgical excision of the node indicated.

\section{Problems in the Diagnosis of Metastatic Nodes.(6)}

- Representative sampling - small metastatic deposits in a reactive node is the main cause of false negative report and may be missed even by repeated aspirations.

- Benign epithelial inclusions of salivary gland origin and thyroid gland origin in cervical nodes have been observed. Although a rare occurrence, this possibility should be kept in mind when only a few epithelial cells without obvious malignant features are found in aspirates.
- If an aspirate consists of necrotic material, it may be difficult to decide whether it represents caseous necrosis or tumour necrosis. Respiration should be done from the periphery of the node and examined for cells. Squamous cell carcinoma is particularly proven to undergo liquefactive necrosis.

- There is a risk of mistaking cystic metastasis of a welldifferentiated squamous cell carcinoma for a branchial cyst. Cystic nodes in the neck may also represent metastases from papillary carcinoma of thyroid.

- $\quad$ Follicle cell centre lymphoma (A type of Non-Hodgkin's lymphoma) may resemble metastasis of small cell anaplastic carcinoma. Also large cell lymphoma can be difficult to distinguish from large cell anaplastic carcinoma without recourse to immunocytochemistry or electron microscopy.

\section{Lymphoma}

- Diagnostic sensitivity has been found to be lower for lymphoma than for metastatic malignancy. For a diagnosis of lymphoma to be of clinical practical value, it must identify good and bad prognostic subgroups and therefore subtype according to one of the current classifications. Cytology is not reliable in this respect.(5)

\section{Problems in Diagnosis of Hodgkin's Disease}

- Poor biopsy field is a problem in the nodular sclerosis subtype. Use of Rotex screen needle may be necessary to obtain sufficient material.

- Reed-Sternberg look alike cells may be present in other conditions like infectious mononucleosis and angioimmunoblastic lymphadenopathy.

- The most difficult differential diagnosis is Timmunoblastic lymphoma in which both the giant cells and background of predominantly small $\mathrm{T}$ cells may be very similar to Hodgkin's. Finally, an occasional example of large.

- Malignant cells with multilobulated nuclei in a background of reactive lymphoid cells representing malignant cells of metastatic carcinoma, which were misdiagnosed as Hodgkin's lymphoma.

- Clusters of epithelioid histiocytes are sometimes seen in smears of Hodgkin's and Non-Hodgkin's and could suggest granulomatous adenitis. The lymphoid cells must always be carefully scrutinised in lymph node smears containing epithelioid cells.

\section{Problems in the Diagnosis of Non-Hodgkin's Lymphoma}

- Cytological subtyping requires experience and expert smear preparation. Smears of a cell suspension prepared in a cytocentrifuge and very helpful in the diagnosis and classification and are better suited for immune marker studies.(7)

- $\quad$ Smears may be mistaken for reactive lymphadenopathy unless close attention is paid to the finer cytological details. Pseudoglandular clusters may simulate small cell anaplastic or adenocarcinoma. This problem occurs in follicular lymphomas. Immunoperoxidase staining for cytokeratin and panleucocyte marker can solve the problem of distinguishing between lymphoma and small cell carcinoma. 


\section{Granulomatous Lymphadenitis}

- The commonest cause in our set-up is tuberculosis. As seen in this series, tuberculosis can be diagnosed with a reasonable degree of accuracy.(8)

\section{Primary Made Out}

- $\quad$ Staging and treatment accordingly.

- TNM Staging - T stage defined by size in oral cavity, oropharynx and major salivary glands T-stage depends on extent of local involvement in larynx, hypopharynx and nasopharynx.

- Oral cancer $-2 \mathrm{~cm}$ : T1 2 to $4 \mathrm{~cm}$ : T2 >4 cm: T3-T4 adjacent invasion through cortical bone and nearby structures in lip and oral cavity.

\section{Unknown Primary}

- Ipsilateral biopsies of suspicious areas.

- Posterior third of tongue.

- Fossa of Rosenmuller.

- Pyriform sinus.

- Cricopharyngeal area.

- If primary is detected - stage and treat accordingly.

\section{Occult Primary}

- If all these efforts do not detect primary lesion, then it is called as occult primary.

- Treat the nodes by surgery if possible and follow with radiotherapy to all potential primary sites including nasopharynx, base of tongue and the pyriform sinus as well as the neck.

- Regular follow-up of the patient usually reveals primary within 3 months.

- Management of neck secondaries with occult primary.

- The unknown primary carcinoma in the head and neck has been estimated to represent up to $7 \%$ of all head and neck carcinomas.

- Good prognosis with possibility of cure in SCC of head and neck.

- Warrants aggressive treatment.

- Based on the histology.

- Squamous cell carcinoma.

- Lymphoma.

- Thyroid Ca.

- Melanoma.

\section{Proforma}

- $\quad$ Name: Age/Sex: IP/OP: Date:

- Level of enlarged lymph node: Duration:

- Fever - Yes/No. Pain - Yes/No.

- History of contact with TB - Yes/No

\section{Examination of Cervical Lymph Node}

- Number-Single/Multiple

- Tenderness-Yes/No

- Surface-Smooth/Nodular

- Consistency-Soft/Firm/Hard

- Occult Primary-Known/Unknown

\section{Examination of Drainage Area}

- Primary focus of infection - Yes/No

- Malignant Growth-Yes/No

- FNAC -

\section{Open Biopsy}

Comparative study of FNAC/biopsy FNAC-false positive in \% false negative in $\%$.

\section{CONCLUSION}

- Fine needle aspiration forms one of the most important investigations in the initial evaluation of cervical adenopathy. ${ }^{(9)}$

- It is a very simple, uncomplicated outpatient procedure that offers a rapid and specific diagnosis with little trauma and is very cost effective 32 . One of its major advantages is the early confirmation of a malignant disease facilitating institution of immediate treatment.

- It cannot be over-emphasized that fine-needle aspiration is always part of the workup and not the final diagnosis. If the findings do not correlate with the clinical suspicion, weight is given to the clinical picture and diagnostic work-up appropriate for the suspected disease is performed.

- It should be borne in mind that a negative result on the fine-needle aspiration does not rule out malignancy. Proper use of fine needle aspiration requires close communication between an experienced cytologist and surgeon. This series demonstrates that fine needle aspiration is a safe accurate and valuable tool in the evaluation of cervical adenopathy.

- Final analysis shows that HPE is gold standard.(10) for evaluation of cervical node in neck for accuracy in approaching $100 \%$ FNAC analysis shows that accuracy is $95 \%$, since HPE diagnosis is invasive procedure. FNA is better alternate to HPE for malignancy and occult primary for lymph node and benign lesion it is complimentary. So we recommend that reliable supplement, however, role of HPE is absolute.

\section{REFERENCES}

1. Hafez NH, Tahoun NS. Reliability of fine needle aspiration cytology (FNAC) as a diagnostic tool in cases of cervical lymphadenopathy. Journal of the Egyptian National Cancer Institute 2011;23(3):105-14.

2. Das DK. Fine-needle aspiration cytology in the diagnosis of tuberculous lesions. Laboratory Medicine 2000;31(11):625-32.

3. Prasad RR, Narasimhan R, Sankaran V, et al. Fine-needle aspiration cytology in the diagnosis of superficial lymphadenopathy: an analysis of 2,418 cases. Diagn Cytopathol 1996;15(5):382-6.

4. Martin HE, Ellis EB. Biopsy by needle puncture and aspiration. Ann Surg 1930;92(2):169-81.

5. Hay A, Pai I, Pitkin L, et al. Value of fine needle aspiration cytology in head and neck lymphoma: experience in a head and neck cancer unit in the united kingdom. Acta Oto-Laryngologica 2011;131(11):1226-31.

6. Engzell U, Jakobsson PÅ, Sigurdson $\AA$, et al. Aspiration biopsy of metastatic carcinoma in lymph nodes of the neck: a review of 1101 consecutive cases. Acta OtoLaryngologica 1971;72(1-6):138-47.

7. Daskalopoulou D, Harhalakis N, Maouni N, et al. Fine needle aspiration cytology of non-hodgkin's lymphomas. A morphologic and immunophenotypic study. Acta Cytol 1994;39(2):180-6. 
8. Lau SK, Wei WI, Hsu C, et al. Efficacy of fine needle aspiration cytology in the diagnosis of tuberculous cervical lymphadenopathy. The Journal of Laryngology \& Otology 1990;104(1):24-7.

9. Gupta AK, Nayar M, Chandra M. Reliability and limitations of fine needle aspiration cytology of lymphadenopathies. An analysis of 1,261 cases. Acta Cytol 1990;35(6):77783.
10. Singh KK, Muralidhar M, Kumar A, et al. Comparison of in house polymerase chain reaction with conventional techniques for the detection of mycobacterium tuberculosis DNA in granulomatous lymphadenopathy. J Clin Pathol 2000;53(5):355-61. 